\title{
Ameloblastic fibro-odontoma: A case report
}

\author{
Ana-Sueli-Rodrigues Cavalcante ${ }^{1}$, Ana-Lia Anbinder ${ }^{2}$, Nívea-Cristina-Sena Costa ${ }^{3}$, José-Roberto Sá Lima ${ }^{4}$, \\ Yasmin-Rodarte Carvalho ${ }^{5}$
}

${ }^{1} \mathrm{PhD}$, Associate Professor, Department of Bioscience and Oral Diagnosis, School of Dentistry of São José dos Campos. UNESPUniversidade Estadual Paulista, São José dos Campos SP Brazil

${ }^{2} \mathrm{PhD}$, Assistant Professor, Department of Dentistry, Taubaté University, Taubaté SP Brazil. Volunteer Professor, Department of Bioscience and Oral Diagnosis, School of Dentistry of São José dos Campos. São Paulo State University. UNESP, São José dos Campos SP Brazil

${ }^{3}$ Post-graduate Student, Departament of Bioscience and Oral Diagnosis, School of Dentistry of São José dos Campos. UNESPUniversidade Estadual Paulista, São José dos Campos SP Brazil

${ }^{4}$ PhD, Full Professor, Department of Diagnosis and Surgery, School of Dentistry of São José dos Campos. UNESP- Universidade Estadual Paulista, São José dos Campos SP Brazil

${ }^{5} \mathrm{PhD}$, Full Professor, Department of Bioscience and Oral Diagnosis, School of Dentistry of São José dos Campos. UNESPUniversidade Estadual Paulista, São José dos Campos SP Brazil

Correspondence:

Av. Engenheiro Francisco José Longo, 777

Jd. São Dimas. São José dos Campos - SP

Brazil CEP: 12245-000

anasueli@fosjc.unesp.br

Cavalcante ASR, Anbinder AL, Costa NCS, Lima JRS, Carvalho YR. Ameloblastic fibro-odontoma: A case report. Med Oral Patol Oral Cir Bucal. 2009 Dec 1;14 (12):e650-3.

http://www.medicinaoral.com/medoralfree01/v14i12/medoralv14i12p650.pdf

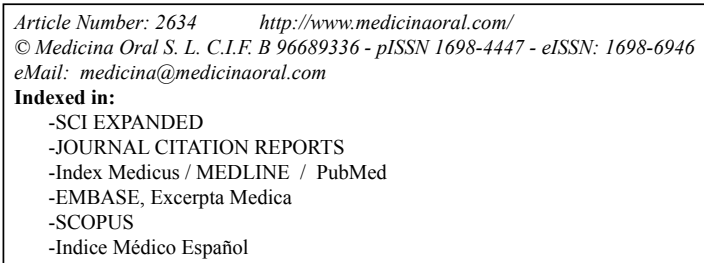

\begin{abstract}
Ameloblastic fibro-odontoma (AFO) is a rare, benign, slow-growing odontogenic tumor, generally asymptomatic and more prevalent in children and adolescents. We report a case of AFO in the mandible of an eight-yearold Caucasian male patient, and review the literature. Intraoral examination revealed a swelling extending from the deciduous second molar to the retromolar triangle, covered with normal mucosa. A panoramic radiograph showed a large, well-demarcated radiolucency with radiopaque areas. The provisional diagnosis was of AFO, and so an incisional biopsy was performed. Histologically, the lesion was composed of connective tissue resembling the dental papilla, with epithelial strands or islands, as well as denticles and amorphous masses of enamel and dentin consistent with a diagnosis of AFO. Surgical excision and curettage of the lesion were performed. The patient has been monitored for eight years and the lesion has not recurred.
\end{abstract}

Key words: Odontogenic tumors, odontoma, fibro-odontoma, ameloblastic. 


\section{Introduction}

Odontogenic tumors are a heterogeneous group of diseases ranging from hamartomas to benign and malignant neoplasms with metastatic potential. Odontogenic tumors arise from odontogenic epithelium, ectomesenchyme and mesenchyme (1). Recent studies assessing large numbers of cases have shown that these tumors constitute $0.84 \%$ to $1.78 \%$ of the histopathological findings of renowned oral pathology departments (2-4). Odontogenic tumors account for $7 \%$ of the cases of oral lesions found in children and adolescents (5).

According to the World Health Organization (1), ameloblastic fibro-odontoma (AFO) is a tumor with histological features similar to those of ameloblastic fibroma (AF), but with inductive changes that lead to the formation of dentin or enamel.

Among the odontogenic tumors, the incidence of AFO varies from $0.3 \%$ to $1.7 \%(3-5)$, reaching $4.6 \%$ when only the cases in children are considered (5). Most cases are diagnosed in the patient's first two decades of life, between the ages of nine and eleven years on average $(3,6)$. There is a slightly higher incidence of AFO in males, as well as in the posterior mandible $(3,6)$. AFO is generally an asymptomatic, slow-growing tumor, commonly associated with an unerupted tooth.

Radiographically, ameloblastic fibro-odontoma presents as a well-demarcated radiolucency containing radiopaque areas $(6,7)$. Conservative surgical excision is the treatment of choice (6), and the lesions does not tend to recur (1).

\section{Case Report}

A white, eight-year-old male patient was taken by his mother to the Multidisciplinary Center for Oral Diseases, School of Dentistry of São José dos Campos, São Paulo State University (UNESP), Brazil, complaining of "a ball in the face" (sic) that had appeared nine months before. The mother said that she had taken her son to a dentist as soon as she had noticed the swelling.

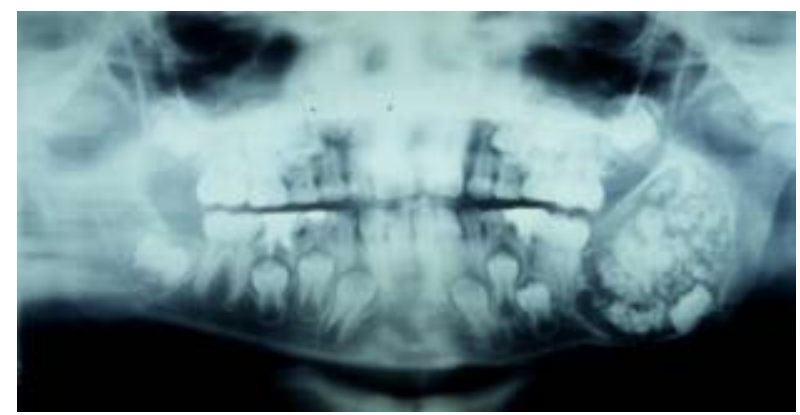

Fig. 1. First panoramic radiograph. Large, well-demarcated radiolucent lesion affecting the angle and part of the ramus of the mandible, containing radiopaque areas of varying size, causing the displacement of the tooth germ of the lower second molar.
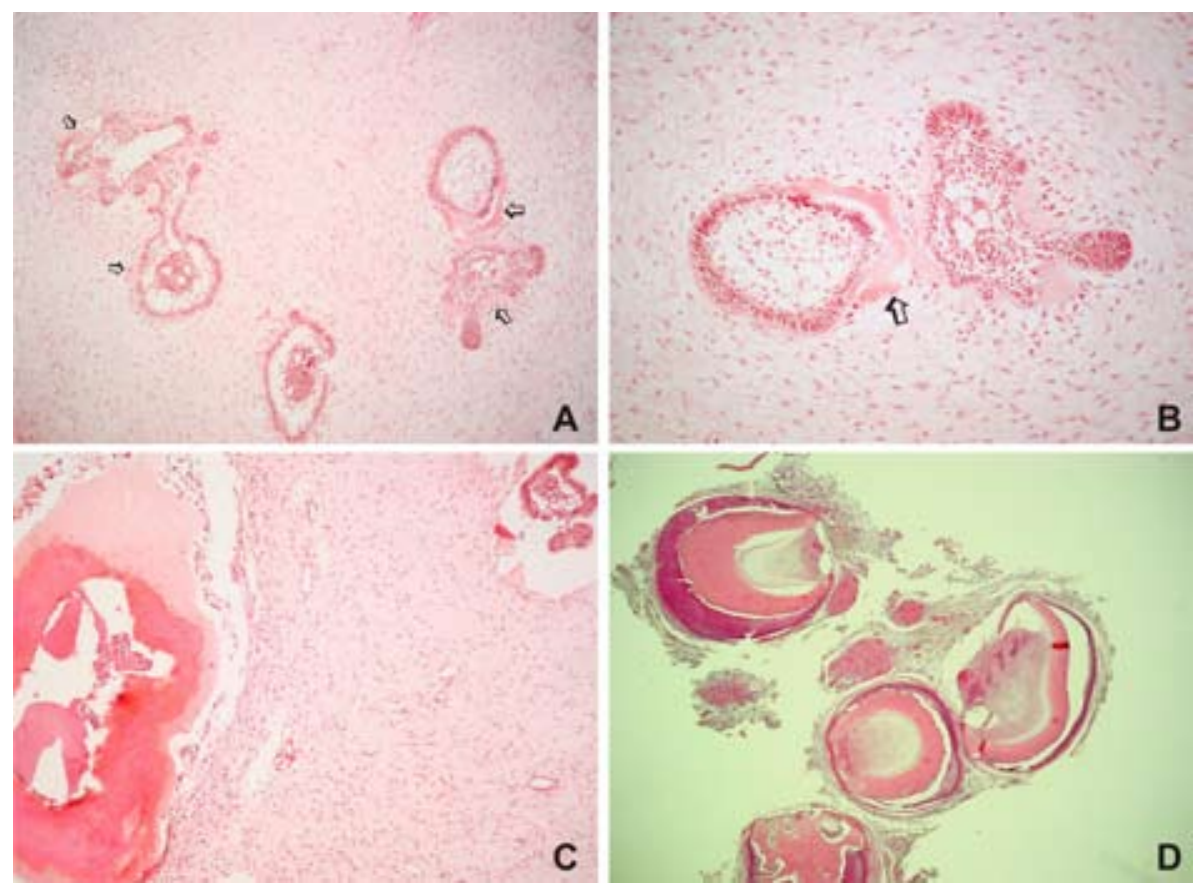

Fig. 2. Microscopic appearance of the lesion (Hematoxylin and eosin). A. Islands of odontogenic epithelium with peripheral cells resembling ameloblasts and central cells resembling the stellate reticulum of the enamel organ amid loose connective tissue, similar to the dental papilla. Near the epithelial cells, it is possible to observe dental hard tissue formation (arrows-original magnification X100). B. High power microscopic view showing an epithelial island amid loose connective tissue, similar to the dental papilla, and dental hard tissue formation (arrows-original magnification X200). C. Calcified dental tissues are found on the left. On the upper right, an epithelial island can be observed (original magnification X100). D. Microscopic appearance of the decalcified hard tissue removed from the lesion. Denticles with crown composed of enamel matrix, tubular dentin and pulp tissue (original magnification X25). 
The dentist, suspecting of a lesion of infectious origin, recommended that the boy rinse his mouth with warm water and vinegar. As the swelling did not subside, the mother sought another dentist, who referred the patient to us. The patient's medical history was unremarkable.

Extraoral examination revealed facial asymmetry, with swelling of the left side of the mandible. The swelling was asymptomatic and covered with healthy skin with normal color. Intraoral examination revealed a swelling of the vestibule of mouth, extending from the deciduous second molar to the retromolar triangle, covered with normal mucosa and hard to palpation.

A panoramic radiograph showed a large, well-demarcated radiolucency containing radiopaque areas of irregular shape, affecting the angle and part of the ramus of the mandible. The tooth germ of the lower second molar was displaced to the base of the mandible, whose outer cortex was rather thin (Fig. 1). An occlusal radiograph showed slight swelling of both the buccal and lingual cortical bones. Based on the clinical findings and the diagnostic hypothesis of $\mathrm{AFO}$, an incisional biopsy was performed. Microscopic examination revealed a benign odontogenic tumor with both epithelial and ectomesenchymal components. The ectomesenchymal component comprised loose connective tissue, with abundant extracellular matrix containing young, plump fibroblasts, sometimes with a stellate appearance, with dendritic branches resembling the dental papilla. Amid this tissue there were epithelial cells, either forming double-layer cords or small islands. The cells at the periphery of the epithelial islands were columnar cells, with palisading nuclei and reverse nuclear polarity, similar to ameloblasts. The cells in the middle of the epithelial islands had a stellate reticulum-like appearance, similar to the stellate reticulum of the enamel organ. Near the epithelial cells, dental hard tissue formation could be observed (Fig. 2A-C). Histological sections of the decalcified specimen showed denticles composed of enamel matrix, tubular dentin and pulp tissue (Fig. 2D). Around these structures, reduced enamel epithelium was sometimes observed. In some regions, the dental tissues were disorganized, forming a great mass of dentin around reduced enamel epithelium and enamel matrix.

After a final histological diagnosis of AFO, the patient was submitted to surgical excision and curettage of the lesion, as well as removal of the tooth germ of the second molar. The specimen comprised fragments of soft tissue of elastic or cartilaginous consistency, fragments of hard tissue of irregular shape, and approximately 57 denticles. Histopathological examination of the specimen confirmed the diagnosis of AFO. The patient has been monitored for eight years, and the lesion has not recurred.

Research Ethics Committee of the University approved publication of the case report.

\section{Discussion}

The diagnosis of oral lesions other than caries, periodontitis or periapical diseases has been a challenge for general dentists. Many dentists associate swelling of the jaws with dentoalveolar abscess because of the high incidence of the latter, even when there are no signs of inflammation or in spite of what radiographs show, leading to a wrong diagnosis. This in turn can alter the prognosis of cases that are often easy to solve when a correct, timely diagnosis is made. Occlusal and/or panoramic radiographs are essential to the diagnosis of odontogenic tumors (1) and other intraosseous lesions. The differential diagnosis of a radiolucency containing various amounts of radiopaque foci includes ameloblastic fibro-odontoma and other mixed radiolucent/radiopaque lesions, such as calcifying epithelial odontogenic tumor, calcifying odontogenic cyst, adenomatoid odontogenic tumor and immature stage of odontoma (8). When the anamnesis of a patient reveals that certain epidemiologic factors such as age, typical location of the lesion and typical radiographic findings are met, the diagnosis of ameloblastic fibro-odontoma is easy (8).

According to the World Health Organization (1), AFO is included, along with AF and ameloblastic fibrodentinoma (AFD), in the group of tumors of odontogenic epithelium with odontogenic ectomesenchyme, with or without dental hard tissue formation. The histological features of AF include odontogenic ectomesenchyme that resembles the dental papilla and strands or islands of odontogenic epithelium that resemble the dental lamina and enamel organ. If there is dentin formation, the lesion should be diagnosed as AFD; if there is also enamel formation, it should be diagnosed as AFO (1). Various published studies have investigated this topic $(6,9,10)$. However, the nature of and relationship between the so-called mixed odontogenic tumors (AF, AFD and AFO) and odontomas, which are regarded as hamartomatous lesions (1), are still not clear. It is argued that mixed tumors are in fact neoplasms or yet different stages of a single pathology (11). In this case, AF, AFD and AFO would represent different stages of the same lesion, which would eventually mature and develop into an odontoma. As a matter of fact, at different developmental stages, an odontoma may have histological features of AF, AFD or AFO (9). However, the concept that these lesions represent a continuum of differentiation has been disputed for various reasons, among which lies the fact that patients who are affected by AF are generally older than those affected by AFO (9).

Philipsen et al. (6) raised the hypothesis that AF, AFD and AFO develop in two different ways. The neoplastic lineage would comprise AF and AFD, whereas AFO would be a hamartoma (primary stage of odontoma). Some lesions diagnosed as AFO are probably the immature stage of odontoma. Nevertheless, not all cases 
should be regarded as hamartomas (10), since some lesions show neoplastic behavior (12), and others show malignant features (13). Likewise, most AFs can be considered true neoplasms, but in some cases, especially those occurring during childhood, they may in fact be the immature stage of an odontoma. At present, based on the histological features alone, it is not possible to distinguish an hamartoma from a neoplasm. A small lesion on the occlusal surface of an unerupted molar may be the immature stage of an odontoma, whereas a lesion that causes swelling and cortical expansion is likely to be a true AFO (14). In addition to the clinical features, the patient's age is an important factor to consider when planning the treatment. Thus, the case reported here was diagnosed and treated as AFO.

According to the World Health Organization (1), the distinction between complex and compound odontomas is based on the arrangement of the dental hard tissues found in the lesions. In complex odontomas, these tissues are found in a more or less disorderly pattern, whereas in compound ones denticles are found. However, some authors have suggested that both types of odontoma are pathogenetically different $(6,9)$. A complex odontoma, more commonly found in the posterior mandible, like AFO, could be the end-stage of a hamartomatous lesion. On the other hand, a compound odontoma seldom has any clinical relation with AFO (9) and its occurrence could be the result of local hyperactivity of the dental lamina, leading to hyperdontia $(6,9)$. According to Chen et al.(9), the presence of disorganized odontogenic epithelium and ectomesenchyme, associated with irregular formation of dentin and enamel make it unlikely that structures similar to teeth be formed in AFO. Nevertheless, in the case reported here, denticles were quite apparent.

Because AFO is a well-encapsulated lesion, usually not locally invasive, the recommended treatment is conservative surgical excision (8). Some authors have suggested that the impacted tooth associated with the lesion be preserved (15), while others have reported recurrence after preservation of impacted or retained teeth associated with the lesion (12). In the present case, no recurrence was observed after enucleation of the lesion and extraction of the second molar. Tissue healing was satisfactory, even without the use of bone graft or replacement material.

\section{References}

1. Barnes L, Eveson J, Reichart P, Sidransky D. World Health Organization classification of tumours. Pathology and genetics head and neck tumours. Lyon: IARC Press; 2005.

2. Olgac V, Koseoglu BG, Aksakalli N. Odontogenic tumours in Istanbul: 527 cases. Br J Oral Maxillofac Surg. 2006;44:386-8.

3. Buchner A, Merrell PW, Carpenter WM. Relative frequency of central odontogenic tumors: a study of 1,088 cases from Northern California and comparison to studies from other parts of the world. J Oral Maxillofac Surg. 2006;64:1343-52.

4. Fernandes AM, Duarte EC, Pimenta FJ, Souza LN, Santos VR, Mesquita RA, et al. Odontogenic tumors: a study of 340 cases in a Brazilian population. J Oral Pathol Med. 2005;34:583-7.

5. Guerrisi M, Piloni MJ, Keszler A. Odontogenic tumors in children and adolescents. A 15-year retrospective study in Argentina. Med Oral Patol Oral Cir Bucal. 2007;12:E180-5.

6. Philipsen HP, Reichart PA, Praetorius F. Mixed odontogenic tumours and odontomas. Considerations on interrelationship. Review of the literature and presentation of 134 new cases of odontomas. Oral Oncol. 1997;33:86-99.

7. Oghli AA, Scuto I, Ziegler C, Flechtenmacher C, Hofele C. A large ameloblastic fibro-odontoma of the right mandible. Med Oral Patol Oral Cir Bucal. 2007;12:E34-7.

8. Chang H, Precious DS, Shimizu MS. Ameloblastic fibro-odontoma: a case report. J Can Dent Assoc. 2002;68:243-6.

9. Chen Y, Li TJ, Gao Y, Yu SF. Ameloblastic fibroma and related lesions: a clinicopathologic study with reference to their nature and interrelationship. J Oral Pathol Med. 2005;34:588-95.

10. Takeda Y. Ameloblastic fibroma and related lesions: current pathologic concept. Oral Oncol. 1999;35:535-40.

11. Kramer IR, Pindborg JJ, Shear M. The WHO Histological Typing of Odontogenic Tumours. A commentary on the Second Edition. Cancer. 1992;70:2988-94

12. Furst I, Pharoah M, Phillips J. Recurrence of an ameloblastic fibro-odontoma in a 9-year-old boy. J Oral Maxillofac Surg. 1999;57:620-3.

13. Takeda Y, Kuroda M, Suzuki A. Ameloblastic odontosarcoma (ameloblastic fibro-odontosarcoma) in the mandible. Acta Pathol Jpn. 1990;40:832-7.

14. Miyauchi M, Takata T, Ogawa I, Ito H, Nikai H, Ijuhin N, et al. Immunohistochemical observations on a possible ameloblastic fibroodontoma. J Oral Pathol Med. 1996;25:93-6.

15. Reis SR, De Freitas CE, Do Espírito Santo AR. Management of ameloblastic fibro-odontoma in a 6-year-old girl preserving the associated impacted permanent tooth. J Oral Sci. 2007;49:331-5. 